\section{Ingeniería}

CONTENIDO

Artículos

Análisis numérico de un sistema de aire acondicionado empleando
mallas estructuradas y no estructurades A

Funciones de regresión para caudales extremos en la Vertiente Rojas, Nazareth; Aguilar, José Francisco; Solís, Hernán. Evaluación de daños por agresión ambiental en viviendas
de concreto reforzado. Solís, Rómel; Moreno, Eric; Jiménez, Felipe; Rosas, Victorino.

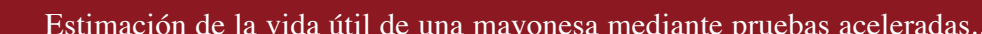
Estimación de la vida útil de una mayonesa
García, Claudia; Molina, Manuel.

Inestabilidad oscilatoria de tensión debido a los motores de inducción …….....................65-75 rde, Gustavo; Araya, Eddie.

Estabilidad y colapso de tensión en sistemas eléctricos Araya, Eddie.

Obtención de pulpa a la soda antraquinona de Tectona grandis creciendo en Costa Rica...........................

Protocolo evaluador para el manejo de desechos y la seguridad ocupacional
aplicado al laboratorio clínico del Hospital San Francisco de Asís de Grecia. Ruiz, Francisco.

9. Euler: su contex
Vargas, Celso.

10. Reserva de resistencia de edificios porticados de concreto armado disenaados conforme al ACI-318/ IBC 2006 ........ Análisis y comentarios

Herrera, Rodolfo.

breve semblanza.

Trabajos de Graduación 2007

Lista de proyectos de graduación de grado y posgrado

Normas

$\sum_{\text {EDTORAL }}$

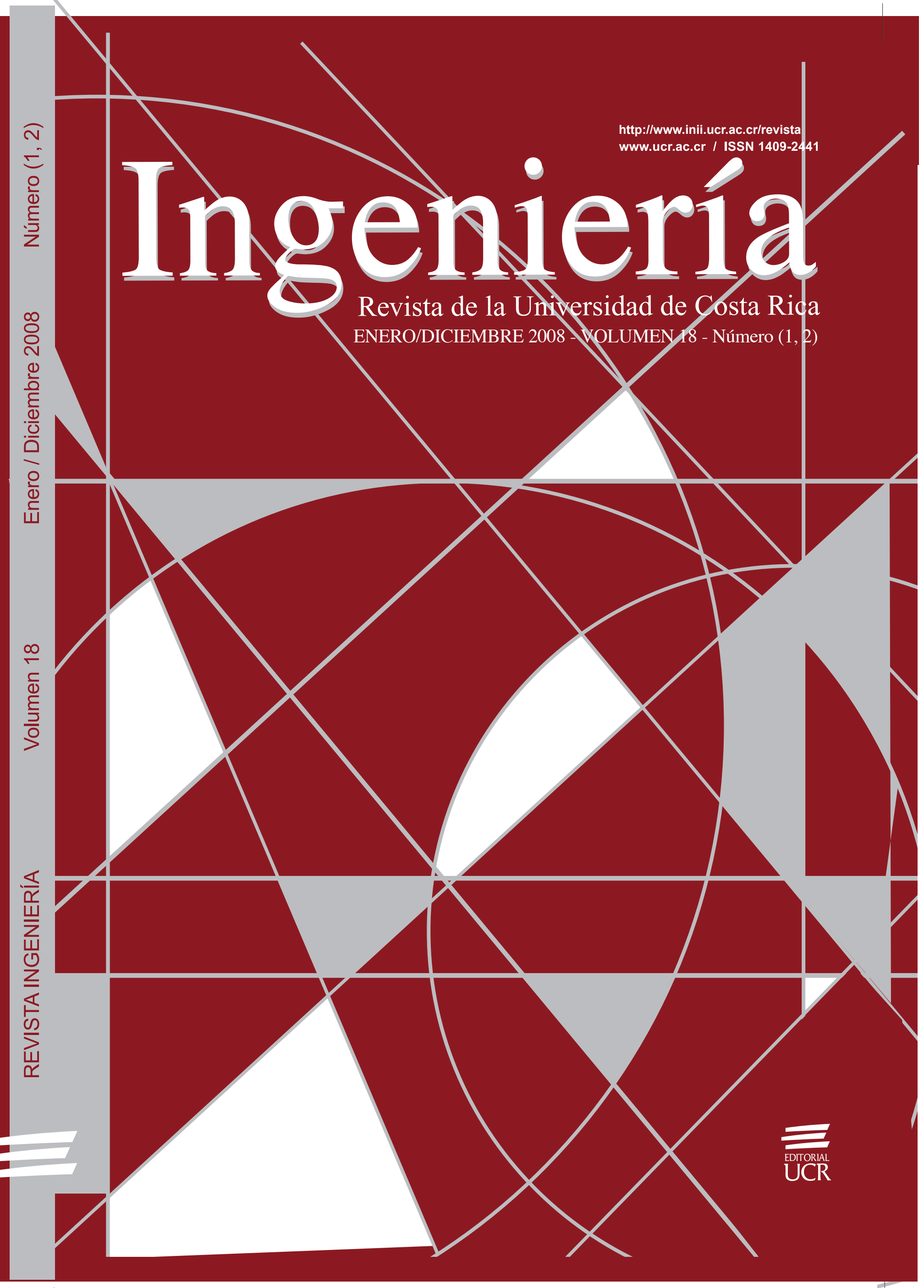




\title{
RESERVA DE RESISTENCIA DE EDIFICIOS PORTICADOS DE CONCRETO ARMADO DISEÑADOS CONFORME AL ACI-318/IBC-2006
}

Juan Carlos Vielma Pérez.

Alex Horia Barbat Barbat Sergio Horacio Oller Martínez.

\begin{abstract}
Resumen
La adecuada capacidad demostrada por los edificios sometidos a la acción de terremotos fuertes ha sido atribuida a la reserva de resistencia de la estructura. Sin embargo, la gran cantidad de factores que contribuyen en la reserva de resistencia, son un obstáculo para que esta haya sido incorporada de forma explícita y transparente en las normas de proyecto sismorresistente. En este artículo se aborda el tema de la reserva de resistencia, para lo que se estudia un conjunto de edificios regulares porticados de concreto armado que han sido proyectados conforme a las prescripciones del ACI318, con las cargas sísmicas prescritas en el IBC-2006. Los resultados muestran que la reserva de resistencia es independiente de la redundancia de los edificios y que los valores recomendados en el IBC-2006 subestiman la capacidad de los edificios con períodos intermedios y largos.
\end{abstract}

Palabras clave: reserva de resistencia, redundancia, análisis no lineal, cortante en la base, proyecto sismorresistente.

\begin{abstract}
The adequate capacity of the buildings during the action of strong earthquakes, have been often attributed to the structural overstrength. However, the large number of factors contributing to increasing the lateral capacity of buildings has avoided that the overstrength was incorporated on an explicit and transparent way in the modern earthquake resistant design codes. This paper focuses the study of the overstrength of regular reinforced concrete framed buildings, designed according to ACI-318, to meet the seismic actions prescribed in the IBC-2006. The results show that the overstrength is virtually independent of the redundancy of the frames and also the values suggested in the IBC-2006 tend to overestimate the capacity of buildings with middle and high periods.
\end{abstract}

Keywords: overstrength, redundancy, non-linear analysis, base shear, seismic design.

Recibido: 8 de diciembre del 2008 • Aprobado: 17 de marzo del 2009

\section{INTRODUCCIÓN}

La supervivencia de edificios a terremotos fuertes suele atribuirse a su capacidad adicional, alcanzada gracias a la contribución de diversos factores. Estos factores intervienen desde la etapa del proyecto hasta la etapa constructiva, mejorando, en muchos casos, las características resistentes del modelo asumido en el análisis. Tradicionalmente se ha definido a la reserva de resistencia de una sección $\lambda_{\theta}$, como la razón que existe entre la resistencia real $S_{0}$ y la resistencia ideal $S_{i}$ para la cual se proyecta dicha sección según la ecuación (1).

$$
\lambda_{0}=\frac{S_{0}}{S_{i}}
$$

En el caso de las secciones de concreto armado, el factor de reserva de resistencia depende, entre otras cosas, de la resistencia real del acero de refuerzo, que suele ser superior a la nominal; del incremento de la resistencia 
debido al endurecimiento (hardening) postplastificación del acero, de la resistencia del concreto, superior a la resistencia nominal debida a la edad del concreto o al efecto beneficioso que tiene el confinamiento sobre la resistencia a compresión, una vez que se han alcanzado ciertas deformaciones en el concreto.

Bertero (1986) y Whittaker, Hart y Rojahn (1999) aceptan que la reserva de resistencia es uno de los componentes del factor de reducción de respuesta $R$ empleado en la determinación de los espectros inelásticos de proyecto a partir de los espectros elásticos. En el ATC-19 (1995) se ha propuesto separar al factor de reducción de respuesta, en tres componentes, mediante la ecuación (2):

$R=\left(R_{S} \cdot R_{\mu}\right) R_{R}$

Donde $R_{S}$ es el factor de reducción por reserva de resistencia, $R_{\mu}$ es el factor de reducción por ductilidad y $R_{R}$ es el factor de reducción por redundancia estructural. De estos tres factores sólo el segundo ha sido estudiado a profundidad (Vielma, Barbat y Oller, 2007).

$\mathrm{Al}$ revisar las normas de proyecto sismorresistente, se observa que la reserva de resistencia no aparece de forma explícita, solo el UBC-97 y el IBC-2006 contienen valores de reserva de resistencia que dependen de la tipología y de los materiales constituyentes de la estructura (Vielma, Barbat y Oller, 2007).
Lo anterior posiblemente se deba a la gran cantidad de factores que intervienen en la reserva de resistencia haciendo que esta sea difícil de cuantificar objetivamente en el ámbito de cada elemento estructural, evitando su incorporación de forma racional en las normas de proyecto sismorresistente, por esta razón es más conveniente considerar tanto el efecto de la reserva de resistencia sobre la respuesta global de la estructura obtenidas a partir de las relaciones de fuerza-deformación, como las producidas por el análisis con empuje incremental (pushover analysis) que se muestran en la Figura 1. En esta figura se define a la reserva de resistencia RS como la relación que existe entre el cortante en la base última $\mathrm{V}_{U}$ y el cortante en la base de proyecto VP, ecuación (3):

$R_{S}=\frac{V_{U}}{V_{P}}$

Diversos autores han publicado valores de la reserva de resistencia de edificios porticados de concreto armado, pudiendo notar cierta dispersión entre dichos valores. En el Cuadro 1 se resumen los valores informados en dichas investigaciones.

El factor de reducción por redundancia ha sido menos estudiado (Vielma, Barbat y Oller, 2007); con frecuencia se le considera erróneamente como un factor cuya contribución sobre la respuesta sismorresistente es superfluo o sobrante (Bertero

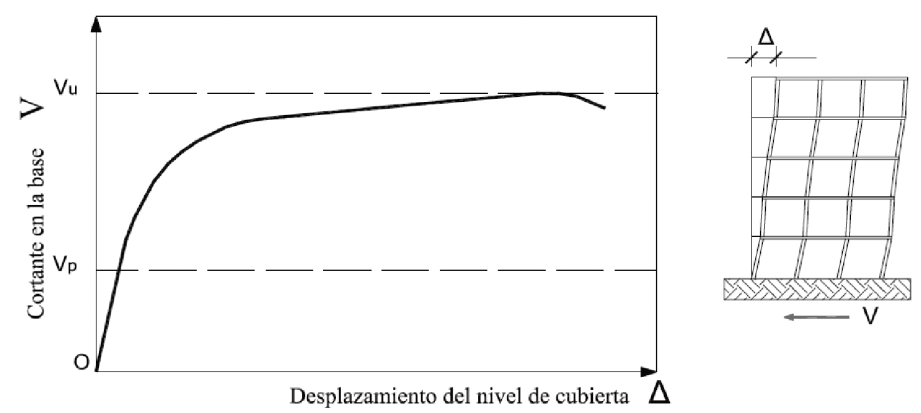

Figura 1. Curva de capacidad típica de un edificio de concreto armado, en la que se muestran el cortante en la base última y el de proyecto.

Fuente: (Los autores). 
Cuadro 1. Resumen de los valores de la reserva de resistencia obtenidos en diversas investigaciones sobre la respuesta de edificios de concreto armado.

\section{Fuente}

Osteraas y Krawlinker (1990)

Uang y Maarouf (1993)

Hwang y Shinozuka (1994)

Fischinger, Fajfar y Vidic (1994)

Panagiotakos y Fardis (1998)

Mwafi y Elnashai (2002)

\author{
Tipología estudiada \\ Pórticos resistentes a momentos \\ Pórticos perimetrales \\ Pórticos con diagonales concéntricos \\ Edificio de concreto de 6 niveles \\ Edificio de concreto de 4 niveles \\ Edificios de concreto de baja y mediana altura \\ Edificios de concreto armado \\ Edificios de concreto de mediana altura con y sin \\ irregularidad vertical \\ Fuente: (Las indicadas internamente).
}

Reserva de
resistencia (RS)
$2,1-6,5$
$1,8-3,5$
$2,2-2,8$
1,9
2,2
$1,6-4,6$
$2,0-2,5$
$2,0-3,0$

y Bertero, 1999). Las normas recientemente han incorporado los efectos beneficiosos de la redundancia (ASCE7-02, UBC-97, IBC2006, EC-08) pero los criterios empleados que consisten esencialmente en la modificación del factor de reducción de respuesta $\mathrm{R}$, son del tipo empírico y no contemplan la posibilidad de que el proyectista pueda modificar o seleccionar adecuadamente el factor de redundancia con base en las características del edificio que se encuentra analizando.

Seguidamente, se presenta la explicación de Humar y Rahgozar (1996), que ilustra la manera como la reserva de resistencia contribuye a mejorar la respuesta de estructuras, cuando estas sobrepasan el límite elástico, y que además permite plantear el procedimiento de cálculo del componente de redundancia dentro del factor de reserva de resistencia.

En la Figura 2 se puede observar un pórtico de un solo vano y de un solo nivel. La viga del pórtico está sometida a la acción de cargas de gravedad constantes y a una carga lateral $F$ que se incrementa progresivamente y que es igual al cortante en la base $V$. Los extremos de los pilares se encuentran apoyados sobre apoyos articulados. El proyecto se ha realizado para un cortante $V_{P}$ procurando que la estructura disponga de pilares más fuertes que la viga, por lo que se espera que en los extremos de estas se formen las rótulas plásticas. Se asume además que el comportamiento de los materiales es elasto-plástico perfecto. Como es de esperar, a medida que la fuerza lateral se incrementa, el desplazamiento lateral del nivel de cubierta de los pilares crece, al principio de manera proporcional obedeciendo al comportamiento elástico, Figura 2a, hasta que el cortante en la base alcanza un valor $V_{Y}$ para el que se produce la plastificación en el extremo izquierdo de la viga, Figura 2b; lo que indica la aparición de la primera rótula plástica. A partir de este momento, la rigidez inicial de la estructura se reduce, aunque mantiene un valor que le permite a la estructura seguir recibiendo incrementos de la fuerza lateral, hasta que se forma una rótula en el extremo derecho de la viga, alcanzándose el valor máximo del cortante en la base $V_{U}$, a partir de la cual el comportamiento se hace completamente plástico. Se define por tanto al factor de reducción por redundancia mediante la ecuación (4):

$$
R_{R}=\frac{V_{U}}{V_{Y}}
$$

Determinar los valores desacoplados del factor de reducción por reserva de resistencia constituye el objetivo principal de esta investigación; para lograrlo se calculan los factores de reserva de resistencia a partir de la respuesta no lineal de edificios de concreto armado, proyectados conforme al ACI-318, para dos niveles de amenaza sísmica (alta y muy alta). Los edificios estudiados son regulares tanto en planta como en elevación, presentando número variable de vanos y de niveles, lo que permite estudiar el efecto de 


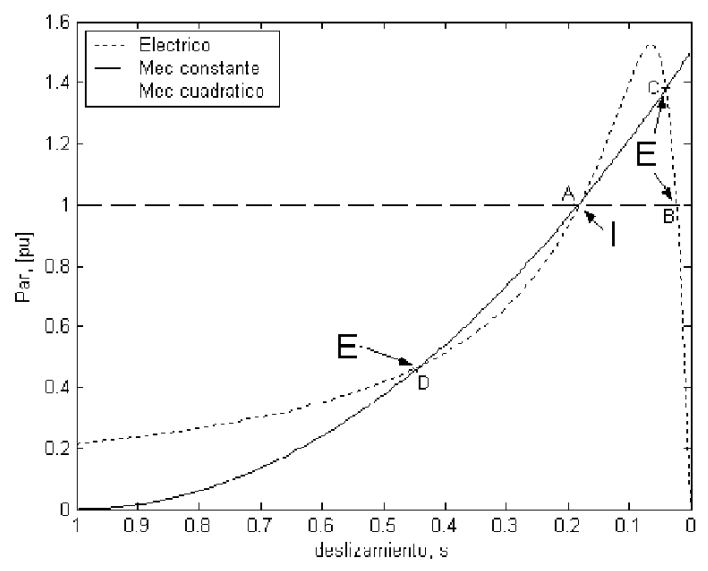

Figura 2. Procedimiento para determinar el factor de redundancia. Fuente: (Los autores).

la redundancia estructural sobre los valores de la reserva de resistencia.

\section{RESPUESTA NO LINEAL DE LOS EDIFICIOS}

La respuesta no lineal de los edificios se obtiene a partir del análisis con empuje incremental aplicado a edificios de concreto armado. Los edificios tienen una geometría que permite clasificarlos como regulares, con un número variable de niveles $(3,6,9$ y 12) y de vanos $(3,4,5$ y 6$)$, ver la Figura 3. Los forjados son de concreto armado con nervios orientados de forma unidireccional, lo que permite definir dos tipos de pórticos: los pórticos de carga (internos y externos) y los pórticos de arriostramiento lateral orientados según la dirección $\mathrm{x}$ e $\mathrm{y}$, respectivamente, ver la Figura 4.

Los edificios han sido proyectados para tener un comportamiento dúctil, enfrentando dos niveles de amenaza sísmica: alta $(0,3 \mathrm{~g})$ y muy alta $(0,4 \mathrm{~g})$. El emplazamiento de los edificios se ha considerado sobre suelos rígidos, suelos tipo B conforme a la clasificación del IBC-2006. En esta norma se contempla un factor de reducción de ocho para pórticos especiales resistentes a momentos.

El detallado de las secciones se ha efectuado siguiendo las directrices del ACI-318, con las consideraciones especiales de confinamiento y ductilidad que quedan determinados a partir de la demanda sísmica. De esta misma demanda queda definido el cortante en la base de proyecto aplicado a cada uno de los pórticos de los edificios, que posteriormente se utilizará en el cálculo del factor de reserva de resistencia.

Una vez que se han proyectado las secciones y se han detallado los elementos de la estructura de los edificios, se procede a la modelización de los mismos aplicando el programa de análisis basado en elementos finitos PLCd (Plastic crack with damage, 1991). En este programa se modelan los elementos de concreto armado aplicando la teoría 


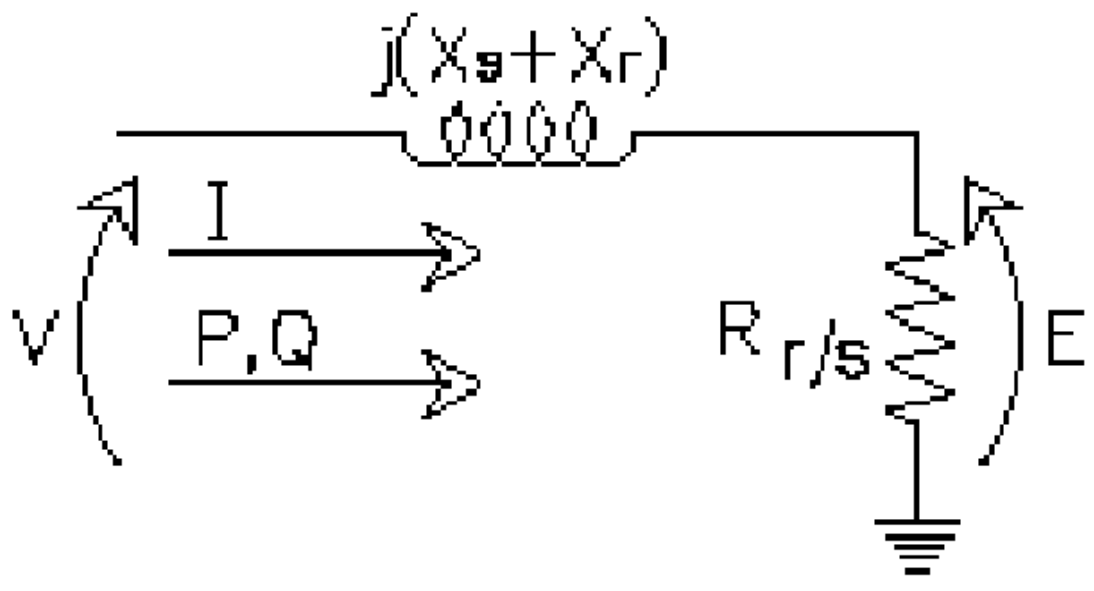

Figura 3. Vista en alzado de los edificios estudiados.

Fuente: (Los autores).
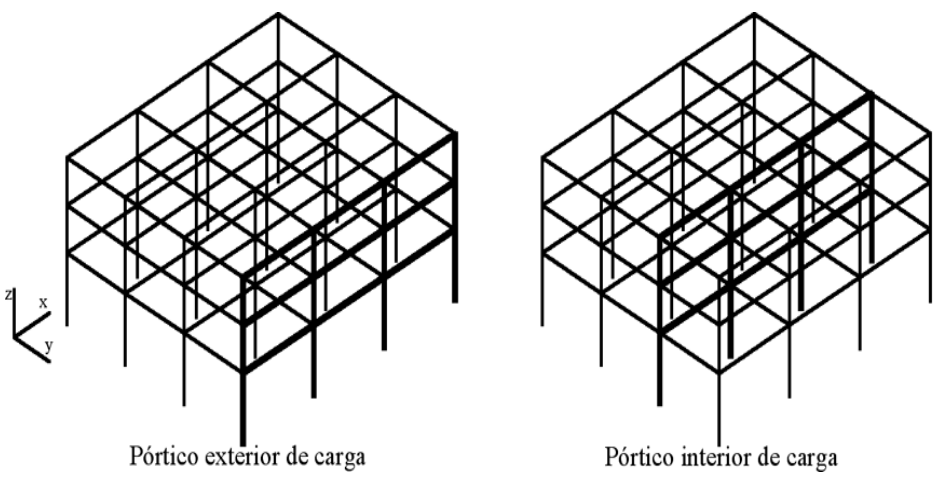

Pórtico interior de carga

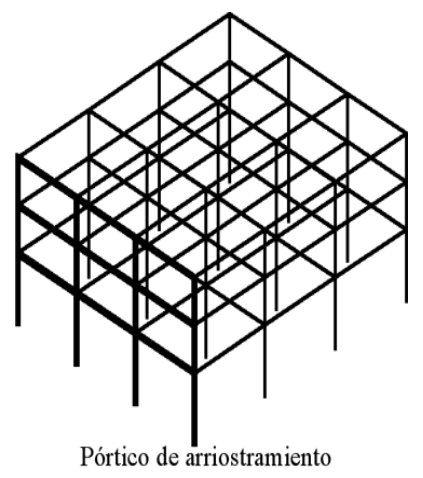

Pórtico de arriostramiento

Figura 4. Ubicación espacial de los pórticos que forman la estructura de los edificios.

Fuente: (Los autores). 
de mezclas, para lo que es necesario discretizar cada elemento en rebanadas, las cuales están constituidas de concreto armado, con diferentes cuantías de refuerzo longitudinal, véase la Figura 5. Allí, los compuestos 1, 2 y 3 corresponden a rebanadas del elemento ubicado en el tramo central de una columna y que poseen diferentes cuantías de acero longitudinal, mientras que los compuestos 4,5 y 6 corresponden a rebanadas del elemento ubicado en las proximidades de la junta viga-columna, que se caracteriza por estar mejor
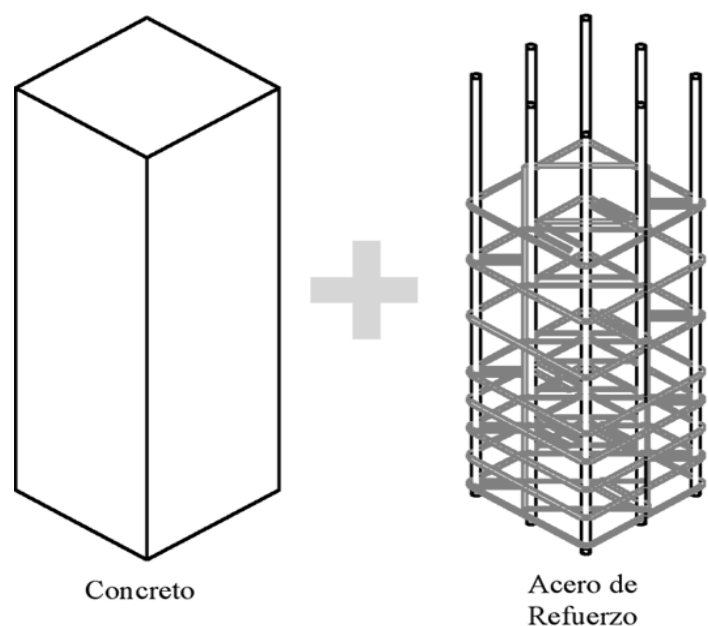

confinada, gracias a un menor espaciado entre los estribos. El efecto del refuerzo transversal se ha considerado por la modificación de la resistencia nominal del concreto, mediante la aplicación del procedimiento formulado por Mander, Priestley y Park (1988).

El paso siguiente consiste en aplicar las cargas a los pórticos para obtener la respuesta no lineal. Primero se aplican las cargas de gravedad, que incluyen el peso propio de la estructura, más las cargas permanentes y variables (por uso) aplicadas

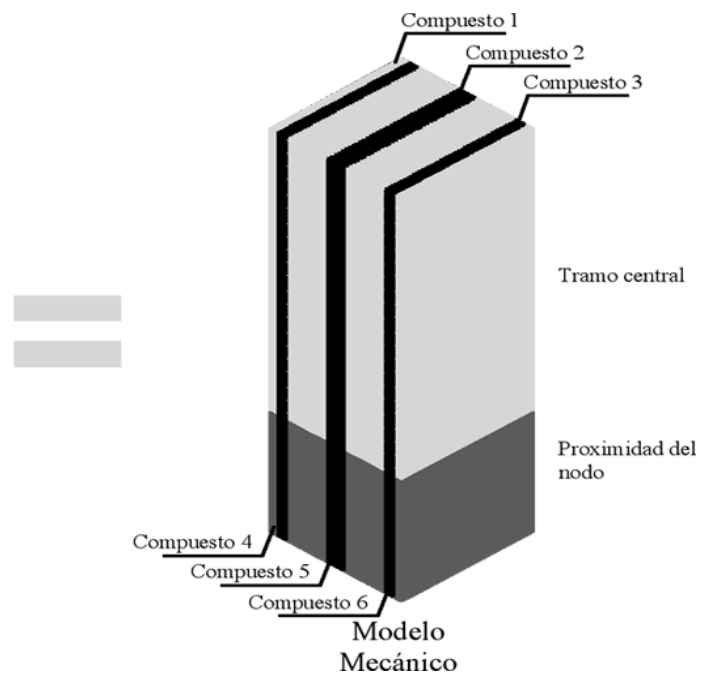

Figura 5. Discretización de los elementos de concreto armado de los pórticos.

Fuente: (Los autores).

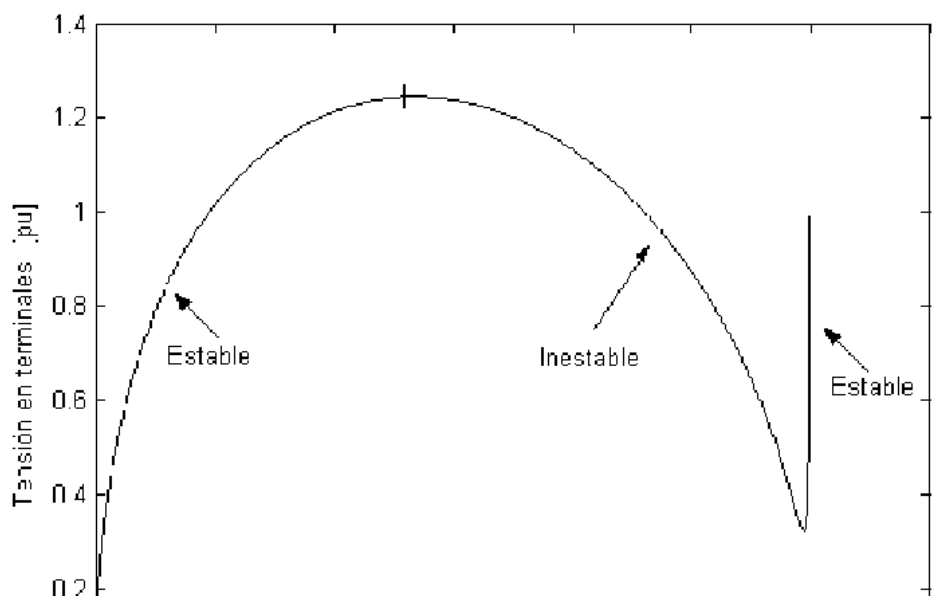

Figura 6. Curvas de capacidad de los pórticos exteriores de los edificios proyectados para $0,4 \mathrm{~g}$.

Fuente: (Los autores). 
Cuadro 2. Valores de la reserva de resistencia de los casos

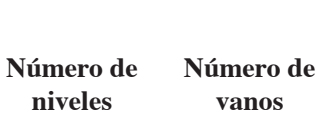

niveles

3

6

9

12

3

6

9

12

3

6

9

12

3

6

9

12 estudiados.

$\begin{array}{ccccc}\begin{array}{c}\text { Pórticos } \\ \text { externos }\end{array} & \text { Pórticos internos } & \begin{array}{c}\text { Pórticos de } \\ \text { arriostramiento }\end{array} & \begin{array}{c}\text { Pórticos } \\ \text { externos }\end{array} & \begin{array}{c}\text { Pórticos internos } \\ 0,3 \text { g }\end{array} \\ 3,39 & 3,21 & 3,93 & 2,38 & 1,88 \\ 2,20 & 2,34 & 2,58 & 2,86 & 1,93 \\ 2,43 & 2,17 & 2,60 & 2,54 & 1,63 \\ 2,23 & 2,04 & 1,97 & 2,43 & 1,82 \\ 4,07 & 3,88 & 5,05 & 2,51 & 1,81 \\ 2,47 & 1,64 & 2,51 & 2,94 & 2,08 \\ 2,38 & 2,04 & 2,34 & 2,23 & 1,52 \\ 2,33 & 2,01 & 1,89 & 2,63 & 1,86 \\ 3,80 & 3,74 & 5,05 & 2,20 & 2,02 \\ 2,23 & 1,81 & 2,63 & 2,99 & 2,07 \\ 2,39 & 2,11 & 2,45 & 2,22 & 1,56 \\ 1,96 & 2,11 & 1,97 & 2,59 & 1,87 \\ 4,54 & 4,65 & 3,93 & 2,48 & 2,02 \\ 1,98 & 2,73 & 2,65 & 3,10 & 2,12 \\ 2,31 & 2,28 & 2,45 & 2,12 & 1,56 \\ 2,26 & 2,05 & 1,89 & 2,49 & 1,80\end{array}$

Fuente: (Los autores).

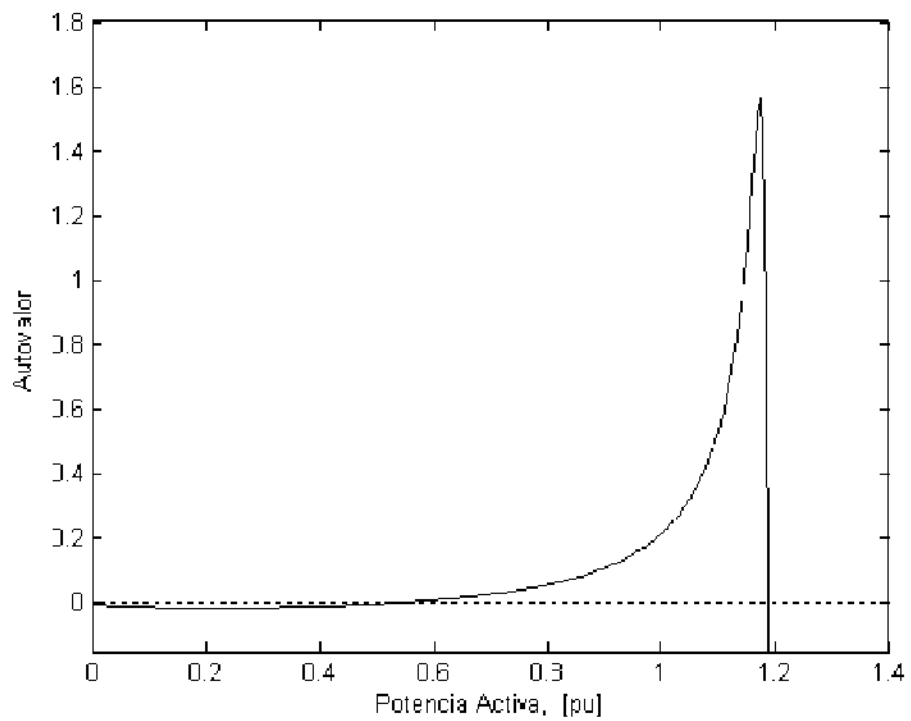

Figura 7. Variación de la reserva de resistencia respecto al número de vanos de los pórticos estudiados: a) Pórticos externos 0,4 g, b) Pórticos internos 0,4 g, c) Pórticos de arriostramiento 0,4 g, d) Pórticos externos 0,3 g e) Pórticos internos 0,3 g.

Fuente: (Los autores). 
en los nodos ubicados en la intersección de las vigas y las columnas. Seguidamente, se aplican las cargas laterales de forma incremental y con un patrón de distribución de fuerzas creciente con la altura, que es una adecuada aproximación a las fuerzas sísmicas equivalentes al primer modo de vibración, predominante en el caso de las estructuras con regularidad tanto en planta como en elevación. Las cargas laterales se aplican hasta que se alcanza el desplazamiento que caracteriza al umbral de colapso del pórtico (Vielma, 2008). En cada incremento de carga se obtiene el desplazamiento lateral del nivel de cubierta del pórtico, correspondiente a un nodo ubicado en el centro de gravedad del nivel citado.

Al graficar la evolución del cortante en la base respecto del desplazamiento del nivel de cubierta se obtiene una gráfica conocida como Curva de
Capacidad, similar a la mostrada en la Figura 1.

\section{RESULTADOS}

El procedimiento expuesto en el apartado anterior ha sido aplicado en la obtención de las curvas de capacidad de los pórticos correspondientes a los edificios estudiados. Es de hacer notar que los pórticos de arriostramiento han sido estudiados únicamente para una aceleración de $0,4 \mathrm{~g}$, por lo que el número total de pórticos estudiados es de ochenta.

Las curvas de capacidad obtenidas del análisis no lineal de los edificios se han graficado normalizando los desplazamientos del nivel de cubierta respecto a la altura total de cada edificio; los valores de los cortantes en la base

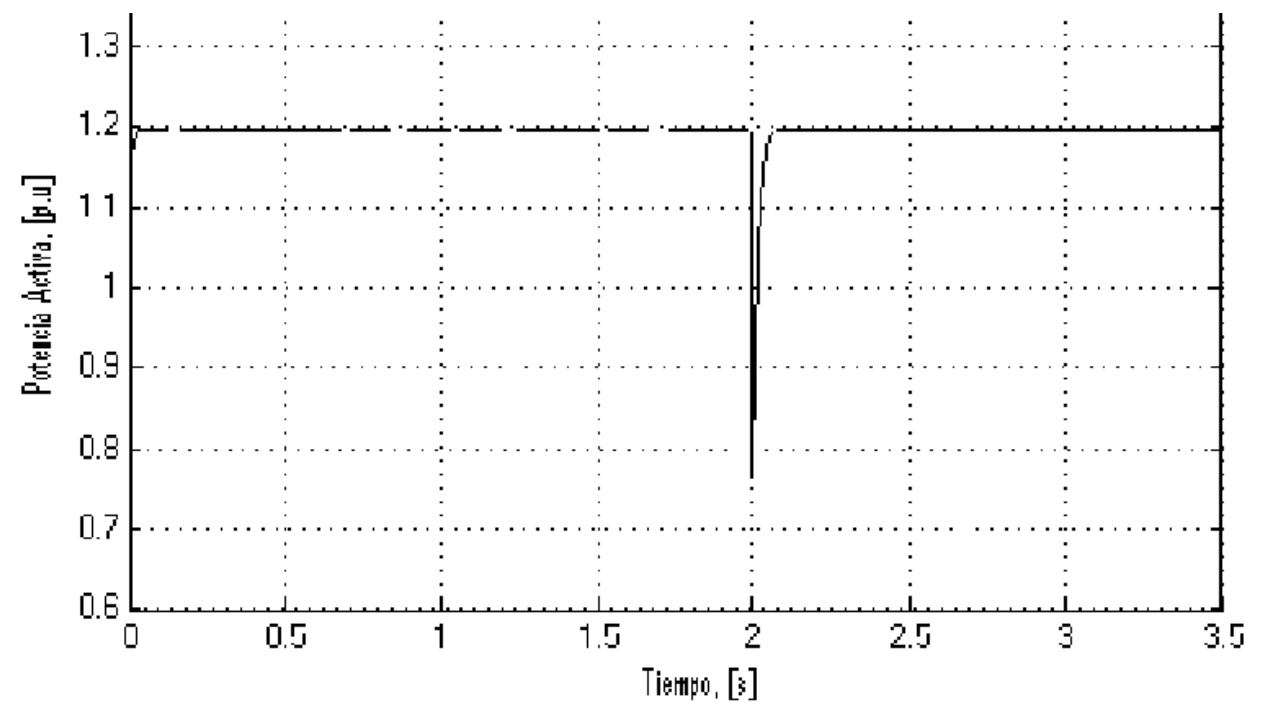

Figura 8. Espectros de reserva de resistencia para a) Pórticos externos $0,4 \mathrm{~g}$, b) Pórticos internos $0,4 \mathrm{~g}$, c) Pórticos de arriostramiento $0,4 \mathrm{~g}, \mathrm{~d}$ ) Pórticos externos $0,3 \mathrm{~g}$ e) Pórticos internos $0,3 \mathrm{~g}$. 
han sido normalizados respecto al peso sísmico de cada edificio, lo que resulta muy conveniente si se desea comparar la respuesta de edificios de diferente número de vanos, pero de similar altura. En la Figura 6, se muestran las curvas de capacidad de los pórticos exteriores de los edificios proyectados para una aceleración básica de $0,4 \mathrm{~g}$.

Los valores de la reserva de resistencia se calculan a partir de las curvas de capacidad aplicando la Ecuación (1). Es evidente que al dividir los valores de las ordenadas entre el cortante normalizado de proyecto correspondiente a cada pórtico, se obtienen valores de la reserva de resistencia que varían respecto al desplazamiento normalizado del nivel de cubierta, por lo que solo se ha considerado el valor máximo de la reserva de resistencia obtenido para cada pórtico. En el Cuadro 2 se muestran los valores máximos de la reserva de resistencia, calculados para los pórticos exteriores, interiores y de arriostramiento de los edificios proyectados para una aceleración básica de $0,4 \mathrm{~g}$ y para los pórticos externos e internos de los edificios proyectados para 0,3 g. Nótese que la reserva de resistencia alcanza valores entre 1,52 y 5,05 y que los pórticos de un mismo edificio presentan diferentes valores de reserva de resistencia, lo que permite sugerir la aplicación de diferentes valores de reserva de resistencia, dependiendo de la relación cargas sísmicas/cargas de gravedad.

Los valores obtenidos para los edificios proyectados para $0,3 \mathrm{~g}$ muestran un comportamiento similar a los de los edificios proyectados para $0,4 \mathrm{~g}$.

Uno de los objetivos de esta investigación es el de estudiar la influencia del número de las líneas verticales resistentes de los pórticos, razón por la que se han analizado edificios con número variable de vanos $(3,4,5$ y 6$)$. En las Figuras $7 \mathrm{a}, 7 \mathrm{~b}$ y $7 \mathrm{c}$ se muestran los resultados para los pórticos exteriores, interiores y de arriostramiento, respectivamente, de los edificios proyectados para amenaza sísmica alta $(0,4 \mathrm{~g})$. Obsérvese que no existe una clara incidencia del incremento del número de vanos en el incremento del valor de la reserva de resistencia, teniendo esta última valores promedios cercanos a 2,5. Iguales resultados se pueden apreciar en las
Figuras $7 \mathrm{~d}$ y $7 \mathrm{e}$, que corresponden a los pórticos exteriores e interiores, respectivamente, de los edificios proyectados para una amenaza sísmica alta $(0,3 \mathrm{~g})$. Para estos grupos de pórticos los valores promedio de la reserva de resistencia se encuentran entre 2 y 2,5 .

Para analizar la influencia de la altura de los edificios sobre la reserva de resistencia, se han graficado los valores de esta respecto al período de los edificios, obteniéndose de esta forma unas curvas para cada tipo de edificio clasificado según su número de vanos. En la Figura 8 se muestran las curvas para todos los casos considerados, en las que se ha colocado el valor recomendado en el IBC-2006 del factor de reserva de resistencia.

De la Figura 8 se observa que los valores calculados del factor de reserva de resistencia dependen del período estructural, en el caso de los edificios proyectados para amenaza sísmica muy alta $(0,4 \mathrm{~g})$. Es notable el incremento de los valores de la reserva de resistencia para el caso de los pórticos de los edificios bajos (tres niveles), que superan al valor recomendado por el IBC2006. Sin embargo, para los edificios medianos y altos, la reserva de resistencia tiene un valor que se aproxima a dos.

\section{CONCLUSIONES}

1. Se presenta un procedimiento general para el cálculo de los factores de reserva de resistencia con base en los resultados del análisis no lineal de edificios.

2. En general, los edificios proyectados conforme al ACI-318, para una demanda sísmica alta o muy alta, exhiben valores adecuados de reserva de resistencia (valores superiores a la unidad).

3. No ha sido posible aplicar un procedimiento objetivo con la finalidad de desacoplar los valores de la reserva de resistencia con los de la redundancia estructural, por lo que se recomienda aplicar ambos factores englobados en un factor de reducción por reserva de resistencia y redundancia en la determinación del factor de reducción de respuesta.

4. El incremento del número de vanos y por tanto el incremento del número de líneas resistentes 
verticales de los pórticos, no incrementa la reserva de resistencia.

5. El nivel de amenaza sísmica tiene influencia sobre los valores de reserva de resistencia. En el caso particular de los edificios proyectados para una amenaza sísmica muy alta, los factores de reserva de resistencia alcanzan valores significativamente mayores para el caso de edificios bajos. Para edificios de mayor altura, la reserva de resistencia alcanza valores en torno a dos.

6. Los edificios bajos proyectados para una amenaza sísmica alta, presentan valores de reserva de resistencia menores, en comparación con los proyectados para una amenaza sísmica muy alta.

7. Los pórticos que forman un edificio pueden alcanzar diferentes valores de reserva de resistencia. Se recomienda por tanto aplicar diferentes valores de reserva de resistencia y redundancia, dependiendo de la relación cargas sísmica/cargas de gravedad.

8. Excepto en el caso de los edificios bajos proyectados para una amenaza sísmica muy alta, ninguno de los pórticos de los casos estudiados alcanzó el valor de la reserva de resistencia de tres, propuesto en el IBC-2006.

\section{REFERENCIAS BIBLIOGRÁFICAS}

ACI Comitee 318. (2005). Building code requirements for structural concrete ACI 318-05. Farmington Hills, Michigan.

Applied Technology Council. (1995). Structural response modification factors. ATC-19. Redwood City, California.

Balendra, T. \& Huang, X. (2003). Overstrength and ductility factors for steel frames designed according to BS5950. Journal of Structural Engineering, 8(129), 1019-1035.

Barbat, A. H.; Oller, S. \& Vielma, J. C. (2005). Cálculo y diseño sismorresistente de edificios. Aplicación de la norma NCSE-02. Monografía CIMNE IS-56, Monografías de ingeniería sísmica. Barcelona.
Barbat, A. H.; Oller, S. \& Vielma, J. C. (2007). Confinamiento y ductilidad de los edificios de concreto armado. Serie de monografías ARCER, monografía № 5, Madrid.

Barbat, A. H.; Vielma J. C. \& Oller, S. (2007). Seismic safety of the limited ductility buildings existing in Spain, Invited Lecture on Sismica $2007,7^{\circ}$ Congresso de Sismologia e Engenheria Sismica, Oporto, Portugal.

Bertero, R. \& Bertero, V. (1999). Redundancy in earthquake-resistant design. Journal of Structural Engineering, 11(125), 81-88.

Bertero, V. (1986). Evaluation of response reduction factors recommended by ATC and SEAOC. Proceedings $3^{\text {rd }}$ U.S. National Conference on Earthquake Engineering. Earthquake Engineering Research Institute. Oakland. 1663-1673.

Fischinger, M.; Fajfar, P. \& Vidic, T. (1994). Factors contributing to the response reduction. $5^{\text {th }}$ US NCEE. Chicago. 97-106.

Hwang, H. \& Shinozuka, M. (1994). Effect of large earthquake on the design of buildings in eastern United States. Proceedings 5th U.S. National Conference on Earthquake Engineering. Earthquake Engineering Research Institute. Oakland. 223-231.

International Code Council. (1995). International building code-2006 (IBC-2006). Cincinnati, Ohio. 2006.

Jain, S. and Navin, R. Seismic overstrength in reinforced concrete frames. Journal of Structural Engineering, 3(121), 580-584.

Mander, J. B.; Priestley, M. J. N. \& Park, R. (1998). Observed stress-strain behaviour of confined concrete. Journal of Structural Engineering, ASCE, 8(114), 1827-1849.

Mwafi, A. \& Elnashai, A. (2002). Overstrength and force reduction factors of multistory reinforced-concrete buildings. Structural Design of Tall Buildings, 11, 329-351. 
Osteraas, J. D. \& Krawinkler, H. (1990). Strength and ductility considerations on seismic design. Rep 90, The John Blume Earthquake Engineering Center. Stanford University.

Panagiotakos, T. \& Fardis, M. (1998). Effects of column capacity design on earthquake response of reinforcedconcrete buildings. Journal of Structural Engineering, 2(130), 113-145.

Plastic crack with damage manual (PLCd Manual). (1991) Non-linear thermo mechanic finite element oriented to $\mathrm{PhD}$ student education, code developed at CIMNE.

Uang, C. M. \& Maarouf, A. (1993). Safety and economy considerations of UBC seismic force reduction factors. Proceedings of 1993 National Earthquake Conference. Central United States Earthquake Consortium. Memphis. 121-130.

Vielma, J. C. (2008). Caracterización de la respuesta sísmica de edificios de concreto armado mediante la respuesta no lineal. Tesis Doctorals en Xarxa. Barcelona. ISBN: 97884-691-3475-7.

Vielma J. C.; Lobo, W. \& Rivero, P. (2006). Influencia de la distancia epicentral sobre los factores de reducción por ductilidad. Ingeniería, 1(16), 59-79. San José.

Vielma, J. C.; Barbat, A. H. \& Oller, S. (2007). Comparación entre los factores de reducción de respuesta de la norma NCSE-
02 y del Eurocódigo 8. Acero, 246, 79-95. Madrid.

Whittaker, A.; Hart, G. \& Rojahn, Ch. (1999). Seismic response modification factors. Journal of Structural Engineering, 4(125), 438-444.

\section{SOBRE LOS AUTORES}

\section{Juan Carlos Vielma Pérez}

Doctor. Ingeniero.

Profesor Agregado de la Universidad Centroccidental Lisandro Alvarado, Venezuela. Decanato de Ingeniería Civil.

Teléfono: (58) 2512592135

Facsímil: (58) 2512592166

Correo electrónico: jcvielma@ucla.edu.ve; jcvielma@cimne.upc.edu

\section{Alex Horia Barbat Barbat}

Doctor. Ingeniero.

Catedrático de la Universidad Politécnica de Cataluña UPC, España.

Teléfono: (34) 934016496

Facsímil: (34) 934011048

Correo electrónico: alex.barbat@upc.edu

\section{Sergio Horacio Oller Martínez}

Doctor. Ingeniero.

Catedrático de la Universidad Politécnica de Cataluña UPC, España.

Teléfono: (34) 934017401

Facsímil: (34) 934011048

Correo electrónico: sergio.oller@upc.edu 
